\title{
Currículo de Psicologia, A Psicologia Social e a Formação para a Saúde Coletiva
}

\author{
Curricula of Psychology, Social Psychology and Sus Health Policies \\ Currículo de Psicologia, la Psicologia Social y la Formación para la Salud Colectiva
}

Neuza Maria de Fátima Guareschi ${ }^{1}$

Universidade Federal do Rio Grande do Sul

Thais Bennemann ${ }^{2}$

Grupo Hospitalar Conceição

Gisele Dhein ${ }^{3}$

Universidade do Vale do Taquari

Carolina dos Reis ${ }^{4}$

Universidade Federal do Rio Grande do Sul

Denise Santos Machry ${ }^{5}$

Universidade Federal do Rio Grande do Sul

\section{Resumo}

Este artigo é resultado de uma pesquisa que teve como objetivo visibilizar de que forma os currículos de cursos de Psicologia do Estado do Rio Grande do Sul estão apresentando indicativos de movimento para formar profissionais da saúde para o SUS. Este trabalho foi desenvolvido a partir da análise dos programas das disciplinas dos currículos de seis cursos do Estado do Rio Grande do Sul, destacando e discutindo os direcionamentos tomados pelas ementas, conteúdos e bibliografias e adotando como balizadores os princípios e diretrizes presentes nas políticas de saúde do SUS. O trabalho toma como foco o estudo das disciplinas direcionadas para os conteúdos de Psicologia Social. A partir da análise que realizamos, apontamos para a necessidade de problematizar a construção do sujeito psicólogo, profissional da saúde que os currículos de Psicologia vêm produzindo, e os efeitos que podem vir a operar no cotidiano dos serviços de saúde.

Palavras-Chave: Graduação em Psicologia, Formação de Profissionais de Saúde, SUS

\begin{abstract}
This paper has stemmed from a research aiming at showing how curricula of Psychology courses in Rio Grande do Sul have shown signs of moving towards education of health professionals for SUS. This work has been developed from the analysis of the program of academic subjects, both highlighting and discussing the directions provided by programs, contents and bibliographies, having the principles and guidelines of SUS health policies as its axis. The paper has a special focus on the study of academic subjects that approach the contents of Social Psychology. From the analysis, we have pointed out the need for problematizing the construction of the psychologist subject, a health professional that has been produced by Psychology courses, as well as the effects that might be felt in the daily routine of health services.

Keywords: Undergraduate course in Psychology, Social Psychology, Health Professionals Education, SUS
\end{abstract}

\section{Resumen}

Este artículo resulta de una investigación que ha tenido por objetivo visibilizar de que forma los currículos de los cursos de Psicologia del Estado del Rio Grande do Sul están apresentando indicativos de movimiento para formar profesionales de la salud para el SUS. Este trabajo se ha desarrollado a partir del análisis de los programas de las disciplinas academicas de los currículos de seis cursos del Estado del Rio Grande do Sul, destacando e discutindo los direccionamientos hechos en los objetivos, contenidos e bibliografias; se adotan como balisadores los principios y las directrizes presentes en las politicas de la salud del SUS. El trabajo toma en su foco lo estudio de las disciplinas direccionadas para los contenidos de la psicologia social. A partir de la analisis que realizamos, apontamos para la necesidad de problematizar la construcción de lo sujeto psicológico, profesional de la salud que los currículos de la Psicologia vienen produzindo, e los efectos que pueden venir a operar en el cotidiano de los servicios de la salud.

Palabras Clave: Currículos de Psicologia, Psicologia Social, Salud Colectiva

1 Endereço: Rua General Souza Doca, 270. Cep: 90630-050.

Porto Alegre - RS - Brasil.

2 Endereço: Rua Silva Jardim, 627/309. Cep: 90450-071. Porto Alegre - RS - Brasil

3 Endereço: Rua Guilherme Alves, 901/214. Cep: 90680-001.

Porto Alegre - RS - Brasil.

4 Endereço: Av. Grécia 1100/1205. Cep: 91350-070. Porto Alegre

- RS - Brasil.

5 Endereço: Rua Veranópolis, 805. Cep: 90520-530. Porto Alegre

- RS - Brasil.

\section{Introdução}

A construção do Sistema Único de Saúde (SUS) representou um grande marco de mudança no que se refere à redemocratização no país. As modificações das formas como o SUS passa a abordar as questões da saúde implicam um novo olhar sobre o sujeito, indo além das preocupações sanitárias e 
abrangendo as diversas áreas de vida da população e o próprio exercício da cidadania. Diante desse novo paradigma inaugurado na área da saúde, passam a surgir demandas de mudança de perfis profissionais que possam produzir uma prática condizente com essa perspectiva de cuidado. Dentre esses redimensionamentos, salienta-se a aproximação da Psicologia das necessidades sociais e, em decorrência disso, a inserção dessa área de formação profissional na saúde e para o SUS, conforme proposto pelos Ministérios da Saúde e da Educação no ano de 2005. A partir dessas mudanças, o SUS passa a ser considerado um campo de atuação da Psicologia nos diferentes setores da saúde, e não somente naqueles voltados aos serviços de atenção direta à população. Portanto, abordar o SUS na formação em Psicologia é uma importante forma de inserção da profissão na realidade brasileira e na luta do movimento sanitarista para o desenvolvimento da saúde coletiva.

Nesse sentido, o presente artigo tem como objetivo problematizar a formação em Psicologia, buscando visibilizar a forma com que os conteúdos da área da Psicologia Social e Comunitária, presente nos currículos dos cursos de graduação em Psicologia, podem fundamentar a formação dos profissionais dessa área para o SUS. Analisamos a entrada da Psicologia na saúde coletiva e as contribuições desta para a construção da nova política de saúde através da formação acadêmica. A partir disso, trazemos as discussões produzidas pela pesquisa, que teve por objetivo desenvolver uma análise dos currículos dos cursos de Psicologia de universidades do Rio Grande do Sul no que se refere à demanda de formação de recursos humanos para o SUS, enfocando nesse debate as disciplinas direcionadas para a área da Psicologia Social e Comunitária. Essa discussão é pautada pelas principais ferramentas e conceitos teóricos discutidos na área da saúde coletiva, tais como a ampliação do conceito de saúde, o princípio da integralidade, a política de humanização e a perspectiva do cuidado integral na atenção à saúde. Por fim, apresentamos alguns apontamentos sobre os efeitos que a atual configuração dos currículos disciplinares podem produzir na formação do profissional psicólogo para a área da saúde.

\section{A Formação em Psicologia e as Políticas em Saúde}

No ano de 2005, o Ministério da Educação e o Ministério da Saúde, através da portaria interministerial $n^{\circ}$ 2.118, firmaram parceria para cooperação técnica na formação e desenvolvimento de recursos humanos na área da saúde (Brasil , 2005). Essa associação é resultado de discussões prévias que vêm desde a construção e o planejamento do Sistema Único de Saúde (SUS). A partir da crescente demanda de formação profissional para o SUS e da incorporação da formação para a saúde como uma das ênfases nas Diretrizes Curriculares Nacionais, os cursos de graduação vêm se redimensionando no sentido de formar novos perfis profissionais para atuar nos serviços de atenção à saúde da população brasileira.

A partir da Constituição de 1988, com a implantação do SUS, a concepção de saúde passa a ser entendida como direito do cidadão e dever do Estado como uma questão integral e plural, e não mais como ausência de doença ou de sintomas, mas como uma questão coletiva, e não pública; isso porque o público remete a modelos de programas assistencialistas e a saúde coletiva objetiva a promoção das condições de vida com a participação social. Embora os termos "saúde pública" e "saúde coletiva" sejam empregados muitas vezes como sinônimos, ambos se constituem como campos não-homogêneos. A saúde pública se construiu no final do século XVII, marcando o investimento da medicina nas enfermidades (Birman, 1991); tem como estratégia básica o esquadrinhamento estatístico da epidemiologia no espaço urbano, adotando medidas sanitárias para combater as epidemias. A saúde coletiva, em direção oposta à da saúde pública, "se constituiu através da crítica sistemática do universalismo naturalista do saber médico" (Birman, 1991, p. 9); está intimamente associada à entrada das ciências humanas na saúde, que passam a criticar categorias universalizantes da saúde pública, destacando as dimensões simbólicas, éticas e políticas de forma a relativizar o discurso biológico.

Relacionando-se à saúde coletiva, o Sistema Único de Saúde é orientado por três princípios doutrinários (Brasil, 1990): Universalidade - considera-se que todas as pessoas têm direito de acesso aos serviços de saúde, independentemente de características sociais ou individuais, e é dever do Estado proporcionar isso; Integralidade - compreende-se que cada cidadão é um ser integral submetido às mais diversas situações e deve ser atendido de tal forma, sem compartimentalização das ações de promoção, proteção e recuperação; Equidade - há igualdade entre os cidadãos, e estes devem ser atendidos pelos serviços de saúde conforme suas necessidades. Além de essa política operar com os princípios, estabelece também três diretrizes principais: descentralização - principalmente no que se refere ao primeiro nível de atenção, o que permite às prefeituras uma melhor identificação das necessidades regionais de saúde; atendimento integral - pautado pelo princípio da integralidade; e participação da comunidade - que vai desde a responsabilidade pela saúde individual até a composição do controle social na formulação e vigilância sobre as políticas de saúde. Assim, a saúde passa a ser compreendida como direito do cidadão na promoção das condições de vida e relaciona-se aos modos de ser e estar no mundo, ou seja, saúde como 
um dispositivo dos modos de subjetivação, conforme coloca o artigo 196 da Constituição Brasileira:

A saúde é direito de todos e dever do Estado, garantido mediante políticas sociais e econômicas que visem à redução do risco de doença e de outros agravos e ao acesso universal igualitário às ações e serviços para sua promoção, proteção e recuperação (Brasil, 1988).

Medeiros, Bernardes e Guareschi (2005) enfatizam a importância, para as práticas psicológicas, de entender a saúde como dispositivo na produção de modos de subjetivação a partir do novo conceito de saúde proposto pelo SUS. Essa importância é destacada, uma vez que o conceito de saúde não existiu nas teorias e práticas da Psicologia desde seu início enquanto campo de saber científico. De acordo com as autoras, devemos operar com a ideia de que saúde nas práticas psicológicas não é algo que independe de seu modo de acesso e de significação. Assim, a inserção do profissional de Psicologia nas práticas de saúde coletiva deve tornar-se uma atenção prioritária para a formação nessa área, pois, na história da constituição desse saber como ciência, o conceito de saúde foi sendo integrado às teorias e práticas.

A formação de profissionais na área da saúde, na qual também se encontra a Psicologia, ainda se volta para a abordagem clássica, em que o ensino é tecnicista, preocupado com a sofisticação dos procedimentos e do conhecimento dos equipamentos auxiliares do diagnóstico, tratamento e cuidado, e organizado por áreas de especialidade (Ceccim \& Feuerwerker, 2004). Entretanto, já se observa um movimento nacional no sentido de normatizar a formação e o trabalho dos profissionais da saúde brasileiros, cumprindo a determinação de que a competência para ordenar a formação de recursos humanos na área da saúde é do SUS, conforme o art. 200, inciso III (Brasil, 1988).

É parte desse movimento a criação das Diretrizes Curriculares Nacionais - DCN dos cursos de graduação, em 1997, em decorrência da Lei de Diretrizes e Bases da Educação Nacional - LDB, em oposição ao formato dos currículos mínimos (Brasil, 1996). As DCNs, incluindo o Curso de Psicologia, incentivam a maior flexibilização dos desenhos curriculares, a liberdade para organizar as atividades de ensino e a diversidade das formações pela ampla participação nas realidades locais de saúde e ativa participação estudantil (Ceccim \& Carvalho, 2005). Entre os anos de 2001 e 2004, foram aprovadas as DCNs para os cursos de graduação da área da saúde, firmando nacionalmente que a formação do profissional de saúde deve contemplar o sistema de saúde vigente no país, o trabalho em equipe e a atenção à saúde (Brasil, 2004). A formação em Psicologia define a atenção à saúde como participação na prevenção, promoção, proteção e reabilitação em saúde, segundo referenciais profissionais. A Psicologia definiu a atenção à saúde como seu objetivo específico, além daqueles próprios de um egresso da educação superior.

No ano de 2005, a partir da já citada portaria interministerial, o Ministério da Educação e o Ministério da Saúde ampliaram a abrangência do programa PRÓ-SAÚDE para outros cursos da área, antes restritos aos cursos de Medicina, Odontologia e Enfermagem. Dentre os novos cursos, a Psicologia entra para o desafio colocado à formação de graduar profissionais capazes de promover ações de saúde integral. Ceccim e Feuerwerker (2004) destacam que a integralidade da atenção deve ser norteador das necessidades de mudança na formação dos profissionais dessa área de conhecimento e contribuir à formulação de uma política do SUS para a mudança na graduação das profissões de saúde.

\section{Construção da Psicologia: produção de conhecimento e as práticas psicológicas}

De acordo com Foucault (2002), a Psicologia, a partir do século XIX, passou a preocupar-se fundamentalmente em adequar seu método às ciências da natureza e em encontrar no homem o prolongamento das leis que regem os fenômenos naturais. A metodologia utilizada baseava-se na mensuração, na quantificação e controle dos processos psíquicos. Essa aproximação com o conhecimento do positivismo amparou-se em dois postulados: "Que a verdade do homem está exaurida em seu ser natural e que o caminho de todo o conhecimento científico deve passar pela determinação de relações quantitativas, pela construção de hipóteses e pela verificação experimental" (Foucault, 2002, p.133).

A Psicologia, para obter o status de ciência, inicia seu percurso construindo conhecimento para medir e quantificar o comportamento humano; para isso, produziu medidas, testagens e previsões. Esse movimento faz uma escolha clara pela racionalização dos processos psíquicos, o intuito era a classificação dos indivíduos em modelos predefinidos para se buscar o estado de normalidade e a cura do desvio (Hüning \& Guareschi, 2009).

A formação "psi", em geral, traz certas características modelares, instituídas e bem marcadas; como, em nossa formação, predomina o viés positivista em que se tornam hegemônicos os conceitos de neutralidade, objetividade, cientificidade e tecnicismo e onde, nos diferentes discursos/práticas, o homem, os objetos e o mundo são apresentados como "coisas em si", abstratos, naturais e não produzidos historicamente (Coimbra, 1999, p. 1).

A cientificidade experimentada pela Psicologia situou o psicólogo numa posição de descobridor da realidade psíquica. A objetividade e a neutralidade fizeram com que a Psicologia se preocupasse em buscar a essência psicológica dos sujeitos; tais verdades afastavam a noção de cultura, sociedade e 
contexto histórico da análise (Hüning \& Guareschi, 2009). A Psicologia, como ciência moderna, intervém de forma a criar movimentos de privatização da existência e da experiência. Ao mesmo tempo, operase um movimento de publicização da interioridade psíquica, que deve ser exposta como modo de se curar o cidadão (Medeiros et al., 2005).

$\mathrm{Na}$ década de 1970, nota-se uma conversão considerável de psicólogos para o campo da assistência à saúde pública (Dimenstein, 1998). É principalmente com o movimento de desospitalização e de extensão dos serviços de saúde mental à rede básica de saúde que se dá a inserção do psicólogo na saúde (Spink, 2003). A entrada do psicólogo no setor público de saúde dá-se ao mesmo tempo em que o modelo médico-assistencial privatista se encontra no auge, mas também em franco esgotamento. Criticavase o modelo asilar, e dava-se ênfase à formação de equipes multiprofissionais (Dimenstein, 1998).

Em 1986, a VIII Conferência Nacional de Saúde, evento que definiu as bases do projeto para a construção do Sistema Único de Saúde, serviu para incrementar o processo de ampliação da diversidade de profissionais no campo da saúde (Brasil, 1986). Com esse movimento, pela primeira vez, a saúde deixa de ser concebida como estado biológico abstrato de normalidade ou de ausência de doença e passa a ser concebida como resultado de um conjunto de condições coletivas de existência, como expressão ativa de um direito de cidadania (Dimenstein, 1998). Spink (2003), seguindo essa linha, define a Psicologia Social da Saúde como comprometida com os direitos sociais a partir de uma ótica coletiva, portanto, fugindo das perspectivas tradicionais ou intraindividuais e dialogando com teorias e autores que pensam as formas de vida e de organização na sociedade contemporânea. Assim, a Psicologia Social da Saúde tende a atuar nos serviços de atenção primária, em contextos comunitários, em problemas de saúde em que pesam a prevenção à doença e a promoção à saúde, atuando em conselhos de saúde, comissões e fóruns de elaboração de políticas públicas e saúde coletiva.

Assim, a saúde coletiva constitui-se em um importante campo de atuação do psicólogo na atualidade, no entanto, a formação em Psicologia pouco se orienta para a discussão dos princípios do SUS. Na Psicologia, bem como nas demais profissões da área da saúde, predomina o referencial positivista, centrado no modelo orgânico, voltado para a intervenção assistencial em detrimento da intervenção preventiva e promotora de saúde. A maioria dos cursos continua formando profissionais como se fossem trabalhar nos EUA ou no Brasil dos anos 80 , num modelo de saúde que não é público e que intervém sob o princípio de saúde como ausência de doença (Campos, 2006). A ausência de discussão das políticas públicas de saúde na formação em
Psicologia deve ser colocada em questão por vários motivos:

Primeiro, era (é) a oportunidade de transformações no perfil de formação em Psicologia, rompendo com modelos individualizantes; segundo, tendo em vista as modificações nas demandas de trabalho que vêm ocorrendo, é importante que o psicólogo se prepare já em sua graduação (vale dizer que o público não se restringe ao estatal); terceiro, refletir criticamente sobre as políticas públicas e o saber-agir da Psicologia atende aos anseios de profissionais que já trabalham com essa questão, além do anseio de usuários dos serviços (Bernardes, 2006, p.4).

$\mathrm{Na}$ discussão teórica sobre Psicologia como ciência, formação acadêmica, práticas psicológicas e transformações do conceito de saúde junto às políticas em saúde coletiva, apresenta-se um cenário nacional de revisão dos rumos do fazer-Psicologia e do fazer-saúde em direção ao projeto de consolidação dos ideais da Reforma Sanitária que estão colocados na legislação federal através da criação e institucionalização do SUS.

\section{Os Currículos dos Cursos de Psicologia e a Análise das Disciplinas}

Alguns cursos de Psicologia, para ir ao encontro das ênfases descritas nas DCNs, têm inserido nos currículos mudanças no sentido de contemplar a formação em saúde, tomando como parâmetro a política de formação proposta pelo SUS. Este artigo propõe-se a apresentar uma análise dos currículos de seis cursos de graduação do Estado do Rio Grande do Sul, de duas instituições confessionais, duas federais e duas comunitárias. A escolha dessas universidades se deu pelo fato de esses cursos de Psicologia existirem há mais de dez anos, o que implica que tenham formado profissionais no modelo anterior às Diretrizes Curriculares Nacionais dos Cursos de Psicologia de 2004, evidenciando, assim, um movimento para atender à demanda colocada pelo Ministério da Saúde. Esse movimento também levou em consideração o fato de esses cursos de Psicologia já possuírem egressos em formação nas Residências Multiprofissionais em Saúde (RMS) no Rio Grande do Sul.

O processo de análise aconteceu em três etapas. $\mathrm{Na}$ primeira, realizou-se o mapeamento das grades curriculares dos cursos de Psicologia selecionadas, em que foram identificadas as disciplinas que, a partir da nomenclatura, remetem direta ou indiretamente às temáticas da saúde. Os materiais dessa primeira etapa foram dos programas das disciplinas, retirados dos portais da Internet das universidades.

$\mathrm{Na}$ segunda etapa, realizamos o levantamento e análise de materiais bibliográficos para indicar e situar aspectos teóricos e históricos de como essas disciplinas foram sendo inseridas nos currículos de formação da área. Essa análise teórica e histórica 
possibilitou encontrar alguns pontos para a organização das disciplinas em três áreas do conhecimento: área da Psicologia Social e Comunitária, área da Psicopatologia e Avaliação Psicológica e área das Biomédicas. Como já referido, este artigo trata somente da análise dos programas das disciplinas da área da Psicologia Social e Comunitária. A análise dos programas das disciplinas das outras áreas são aprensentadas em outras publicações.

Assim, na área da Psicologia Social e Comunitária, a análise baseou-se em autores que falam sobre a história desta área, contemplando o movimento histórico da Psicologia Social e Comunitária no Brasil bem como a importância da diversidade e multiplicidade das interlocuções teóricometodológicas desta área como: Silvia Lane, Aroldo Rodrigues, Robert Farr, Pedrinho Guareschi, Rosane Neves da Silva e Neuza Guareschi.

As perspectivas teórico-metodológicas discutidas por esses autores sobre Psicologia Social apontam que esta área tem passado por transformações nos enfoques dos conteúdos e nas metodologias de pesquisa. Até o final dos anos 70, utilizavam-se, em especial, os aportes teóricos de base positivista, e as pesquisas acadêmicas faziam uso, principalmente, de métodos experimentais, visando a adaptação dos indivíduos à sociedade. No entanto, essa perspectiva positivista e de influência norte-americana passou a ser criticada por reproduzir interesses hegemônicos da sociedade e por possuir uma visão universal e ahistórica de ser humano.

Destacamos cinco pontos que consideramos como aqueles que fundamentam os pressupostos da Psicologia Social e Comunitária e que inserem transformações tanto nas produções teóricas e metodológicas quanto nas práticas psicológicas. Tendo como argumento as condições sócio-históricas brasileiras, em um momento em que problemas sociais como violência urbana, desemprego e favelização da população nos grandes centros passam a emergir em decorrência do processo de industrialização brasileira, o primeiro ponto refere-se a uma crise na Psicologia Social nesse período, em decorrência das questões levantadas.

O segundo ponto decorre da demanda de produção de conhecimento a partir da perspectiva da Psicologia Social Crítica, instaurada a partir da crise e inspirada no paradigma epistemológico do Materialismo Histórico Dialético, que fundamenta discussões sobre as possibilidades de transformação das condições econômicas e sociais de vida da população. O terceiro ponto situa-se quando a Psicologia Social Crítica, com uma concepção de sujeito histórico social e entendendo-o como produto e produtor da história rompe com pressupostos metodológicos, como a neutralidade científica do pesquisador e o uso de instrumentos que garantam a separação entre sujeito e objeto, ou seja, a objetividade e a noção de uma verdade científica. Com esses pressupostos, passam a desenvolver-se a pesquisa participante e a pesquisa ação, envolvendo-se diretamente em contextos de vida de populações carentes e em situações econômicas e sociais precarizadas.

Com o avanço dos debates teóricos e metodológicos e com o aumento significativo de produções científicas, bem como com a formação de profissionais por pesquisadores da Psicologia Social Crítica, ressaltamos, no quarto ponto, o efeito dessas ações nos processos sociais e culturais de grupos e organizações populares. As ações empreendidas nesses contextos para a conscientização e transformação dos processos sociais passam a abranger discussões políticas mais amplas na sociedade que visavam à construção do exercício da cidadania através do direito ao acesso à saúde, da educação e de condições de trabalho justas e dignas. Assim, como quinto ponto, indicamos as implicações da Psicologia Social Crítica na construção teórico-metodológica de outros campos de pesquisas que passam a produzir conhecimentos e que irão formar os saberes da Psicologia Comunitária e da Psicologia do Trabalho - esta última para opor-se à Psicologia Industrial/Empresarial/Organizacional, que foca o indivíduo, visando ao bem da organização, e não à produção de subjetividade a partir da relação do trabalho. Já a Psicologia Social Comunitária passa a integrar-se com profissionais de outras áreas no trabalho da saúde pública, alertando para a formação do psicólogo para o trabalho em todos os campos das Políticas Públicas e Sociais, seja no campo da saúde, da educação ou da assistência social.

\section{A Psicologia Social e Comunitária nos} currículos dos cursos de Psicologia: o social como soma de indivíduos

Apresentamos a seguir a análise dos conteúdos dos programas das disciplinas da área de Psicologia Social e Comunitária a partir de três temáticas que consideramos como as que emergiram como centrais para esta discussão: Sistemas teóricos, Intervenções Sociais e Movimentos Sociais.

\section{Sistemas Teóricos}

É importante destacar que muitas das disciplinas da área da Psicologia Social e Comunitária possuem a mesma e abrangente nomenclatura: Psicologia Social; no entanto, estas se diferem a partir do conteúdo apresentado em seus programas. Enquanto alguns destes abordam conteúdos bem específicos como definição de conceitos, outros objetivam trabalhar perspectivas e teorias desta área da Psicologia.

Assim, os programas das disciplinas desta área, de modo geral, objetivam ensinar sistemas teóricos tradicionais - como a Psicanálise, o Behaviorismo, o Cognitivismo, a Gestalt - para fundamentar e abordar historicamente o ensino em Psicologia Social. Dessa 
forma, nas disciplinas introdutórias de Psicologia Social, os conteúdos são apresentados a partir dessas correntes teóricas que são as que também fundamentam a maior parte dos conteúdos das disciplinas das outras duas áreas da pesquisa - áreas das Biomédicas, e área da Avaliação Psicológica e Psicopatologia.

Os conceitos tratados nas disciplinas da área da Psicologia Social e Comunitária, na sua maioria, são no sentido de compreender as influências ou efeitos do meio social no indivíduo. Esta perspectiva de compreensão dos conceitos a partir do indivíduo e não pelas diferentes relações que se estabelecem entre o indivíduo, grupos, instituições e os contextos sóciohistórico-culturais, não está somente nas disciplinas da área da Psicologia Social e Comunitária, mas também nos conceitos trabalhados nas disciplinas dos outros das outras áreas deste estudo. Isto é verificado, por exemplo, no conceito de identidade sendo fundamentado por sistemas teóricos como a Psicanálise e o Comportamentalismo, teorias que estão sendo aplicadas na área de Psicologia Social e Comunitária e que remetem à identidade do indivíduo sem trazer outros conteúdos nas disciplinas que possam relacionar ou integrar a questão da identidade às diferentes identidades culturais e sociais. No entanto, quando este conceito é enfocado por outras teorias de Psicologia Social como a Teoria Crítica, por exemplo, este apresenta uma compreensão de identidade relacionada aos contextos histórico, econômico e cultural.

Também, os conteúdos das disciplinas desta área remetem à construção da Psicologia Social Moderna, desde uma abordagem positivista, passando com maior ênfase pelas abordagens das teorias críticas fundamentadas no Materialismo Histórico-Dialético, até correntes teóricas que se aproximam do enfoque pós-estruturalista. A Psicologia Social Moderna constitui-se, principalmente, a partir de duas disciplinas: a Psicologia e a Sociologia, que mantêm estreita ligação sustentada por diversos teóricos com produções sobre o indivíduo, sociedade e cultura. Estudos nesta área apontam para o que Farr (1999) denomina de forma sociológica e forma psicológica de Psicologia Social: a primeira, com enfoque nos fatores sociais envolvidos na relação indivíduoambiente e, a segunda forma, mais atenta aos aspectos psicológicos do indivíduo quanto aos modos de vida em sociedade.

Assim, é possível destacar que tanto a forma sociológica quanto a forma psicológica de Psicologia Social estão presentes nos conteúdos dos programas das disciplinas de Psicologia Social e Psicologia Comunitária, que apresentam em seus programas conteúdos como a contextualização da Psicologia Social na América Latina e no Brasil, histórico, principais correntes teóricas, processos de socialização, processos de interação, sendo sustentado, principalmente, pelo autor Aroldo Rodrigues.
Em relação à corrente da Psicologia Social Crítica, esta é fundamentada principalmente por autores nacionais que sustentam a aprendizagem pelos enfoques teóricos do Materialismo HistóricoDialético, mas também por abordagens teóricas críticas mais contemporâneas que discutem o contexto sócio-histórico da sociedade brasileira e da América Latina, como Sílvia Lane, Pedrinho Guareschi, Wanderlei Codo, entre outros. Interessante destacar que, tanto a Psicologia Social Moderna quanto a Psicologia Social Crítica, estão sendo abordadas muitas vezes no mesmo tópico da disciplina dando a impressão que não é dado maior desenvolvimento às diferenças epistemológicas de uma e de outra.

Já, o que se poderia nomear como uma abordagem Pós-Estruturalista, se caracteriza pelo caráter problematizador sobre as relações sociais no contemporâneo a partir de teorias e conceitos que enfocam a linguagem, o discurso, as práticas discursivas, a diversidade cultural e os vetores da sociedade atual, como o mundo virtual, globalizado e os efeitos das tecnologias na produção dos modos de vida. Essa perspectiva teórica, nos programas das disciplinas de Psicologia Social e Comunitária, é demonstrada por enfoques que se construíram a partir da Virada Lingüística, Virada Cultural e o Construcionismo Social, e que iniciam com conteúdos do campo dos Estudos Culturais, direcionando-se para abordagens teóricas foucaultianas e pós-modernas.

Os conteúdos abordados por estas perspectivas são alguns daqueles que também são ensinados a partir de outras perspectivas como a Psicologia Social Moderna e a Psicologia Social Crítica. Porém, quando apresentados nos programas das disciplinas desta área, estes conteúdos se diferem pelo modo com que são apresentados, mas principalmente pelas referências bibliográficas que os fundamentam. Por exemplo: o conteúdo sobre "identidade", quando apresentado num viés da Psicologia Social Moderna, é colocado como Identidade, Papéis Sociais, Meio Ambiente; na Psicologia Social Crítica, como Identidade Social, Conscientização e Ideologia; e nas perspectivas que denominamos como pós-estruturalistas, como Identidades Sociais/Culturais, Linguagem, Produção de Subjetividade.

A inclusão desta perspectiva pós-estruturalista, nos programas das disciplinas da área da Psicologia Social e Comunitária indica, pela forma como apresenta os conteúdos a serem trabalhados, uma estratégia para fomentar a problematização do social como uma invenção da Psicologia, privatizando-o como objeto do conhecimento desta área (Silva, 2005).

\section{Intervenção social}

Como intervenção social se denominou os conteúdos propostos nas disciplinas relacionados aos modos como a Psicologia Social se insere nos diferentes contextos, desde pesquisas para produção 
de conhecimento até intervenções a partir de métodos e técnicas psicológicas, individuais ou em grupo, que visam ajuda, colaboração ou transformação social, e, neste caso, algumas delas vinculadas necessariamente à produção do conhecimento.

A partir dos programas das disciplinas da Psicologia Social e Comunitária que abordam a aprendizagem de intervenções enquanto principais ferramentas metodológicas do psicólogo no contexto de trabalho desta área, emergem duas questões: a primeira é o tratamento da temática intervenção de forma mais estrutural, relacionada a metodologias e técnicas, ou seja, o que é, quem faz, onde faz; e a outra é uma questão mais teórica e epistemológica que fundamenta os modos de intervenção em termos de conhecimento, ou seja, os conceitos que norteiam estas práticas. As intervenções, no geral, são fundamentadas por saberes de diferentes campos do conhecimento, atribuindo, assim, um caráter mais multidisciplinar, interdisciplinar ou mesmo transdisciplinar, dependendo do enfoque teórico no qual esta prática se sustenta.

As disciplinas desta área, com enfoque no aprendizado de intervenções e no trabalho do psicólogo em equipes ou de forma individual, enfatizam, muitas vezes, em seus programas, a multidisciplinaridade e a interdisciplinaridade. A multidisciplinaridade e a interdisciplinaridade, enquanto campos de saber, estão postas a partir da proposição da Psicologia se relacionar com outras áreas, cujos profissionais possivelmente integram as equipes de trabalho, a fim de possibilitar uma contextualização, de forma mais ampla, do objeto sobre o qual se está intervindo. Essas questões são evidenciadas, por exemplo, nos programas das disciplinas Psicologia Hospitalar, Psicologia da Saúde, Psicologia e Políticas Públicas, que propõem conteúdos como a relação entre psicólogo, hospital e outros serviços; a prática psicológica no âmbito hospitalar; o papel da Psicologia no Hospital, o trabalho interdisciplinar, a perspectiva da Psicologia e a especificidade de sua contribuição em equipes multidisciplinares.

Segundo Passos (2001) a multidisciplinaridade reside na lógica do "somatório"; isto é, são várias perspectivas que se somam para olhar um mesmo objeto. O que prevalece aqui é a questão que essa interação entre essas ciências se mantém igual, permanecendo na manutenção dos limites identitários das disciplinas. A interdisciplinaridade, por sua vez, reside na lógica da articulação, em que as disciplinas produzem uma zona de intersecção; há a produção de um novo olhar sobre este objeto, mas tal olhar cria uma nova disciplina, de forma que ainda se mantém a lógica disciplinar, apesar da criação de outra. Sucintamente, podemos dizer que na multi e na interdisciplinaridade segue-se com a manutenção dos limites identitários das disciplinas. É no sentido de produzir uma relação diferente com o objeto de conhecimento da ciência que surge a idéia da transdisciplinaridade, em que a relação que se estabelece é a de perturbação, de afetação, de interferência; desse modo, há uma produção de diferença na desestabilização desses limites das disciplinas, pois a lógica da identidade já não opera mais aqui.

Porém, as distintas perspectivas teóricas de outros campos de saber que formam uma possível multidiciplinaridade, se investigadas quanto às bibliografias referenciadas nos programas, apresentam diferentes compreensões sobre um mesmo objeto. Este fator se faz presente também nos conteúdos dos programas. Embora caracterize uma multidisciplinaridade, não é possibilitada uma discussão epistemológica dos diversos campos de saber que constituem esta multidisciplinaridade, expostas pelos conteúdos, para poder propiciar uma compreensão das diversas concepções de sujeito e de realidade destes outros saberes.

Além disso, na maioria das vezes, a multidisciplinaridade e a interdisciplinaridade são tratadas nos programas das disciplinas como sinônimos; porém, algumas vezes são diferenciadas, como no programa da disciplina Introdução às Ciências da Saúde, quando o conhecimento de outra área do saber vem propor uma interrelação mais direta com a Psicologia, com intuito de promover a integração entre ambas e outro entendimento sobre um determinado objeto. Nesta disciplina, o objeto em questão é a temática da Saúde, abordada a partir da Antropologia, da Sociologia e da Política. No entanto, é importante destacar que, mesmo assim, é difícil afirmar somente a partir do programa da disciplina se isto se caracteriza como proposta de interdisciplinaridade ou simplesmente com o propósito de estabelecer uma relação da Psicologia com outros campos do conhecimento.

Diferentemente do programa da disciplina Introdução às Ciências Sociais para a Saúde, que traz a concepção de saúde a partir de outro campo de saber que não o da Psicologia, o programa da disciplina Saúde, através de sua ementa, propõe a discussão das concepções de saúde na contemporaneidade com enfoque interdisciplinar; o que se destaca nos conteúdos desse programa são as concepções de saúde-doença e saúde como processo em construção inter e transdisciplinar, fundamentados em autores contemporâneos que têm problematizado esta temática da saúde pelo viés da saúde coletiva, com autores como Foucault, Goulart e Madel Luz.

Também, as palavras 'multidisciplinaridade' e 'interdisciplinaridade' aparecem nos conteúdos de disciplinas da área Psicologia Social e Comunitária não necessariamente através da proposição do ensino de conteúdos a partir de diferentes campos de saber, mas como um aprendizado na formação do psicólogo para este poder intervir, trabalhar, auxiliar e compor equipes multidisciplinares e interdisciplinares em 
contextos profissionais.

Ainda quanto ao caráter das intervenções, podemos destacar que a transdisciplinaridade é pouco referenciada nos currículos de Psicologia e, assim como a multidisciplinaridade e a interdisciplinaridade, aparece relacionada somente à atuação do psicólogo na área da saúde. A transdisciplinaridade, como possibilidade de desestabilizar as fronteiras identitárias de diferentes saberes (Passos \& Barros, 2000), é mencionada em alguns programas que enfocam a Saúde Coletiva, como a disciplina Saúde, Intervenções em Saúde Coletiva e Saúde Mental Coletiva, a partir do ensino de conteúdos relacionados a estratégias de intervenção e fundamentados em autores como Gastão Wagner Campos, Roseni Pinheiro e Rubem Mattos.

No que se refere às metodologias e técnicas das intervenções, em muitos programas de disciplinas não se encontram conteúdos que remetam diretamente aos modos que fundamentam e instrumentalizam as intervenções, tanto teoricamente quanto metodologicamente. Isso é evidenciado pela disciplina nomeada de Intervenções Psicossociais, cujos conteúdos abordados são: definição; quem realiza; tipos de intervenção; campos de trabalho. Assim, o entendimento de como ocorrem o ensino e aprendizagem sobre questões metodológicas da intervenção, podem ser somente deduzidas a partir das bibliografias apresentadas, situadas na perspectiva da Psicologia Social Crítica.

A forma com que as intervenções e os modos de realizá-las estão postas nos programas das disciplinas são também entendidas como uma metodologia de pesquisa e como estratégia de organização e mobilização nos movimentos sociais, principalmente, aquelas que visam transformações sociais. A abordagem das intervenções como metodologia de pesquisa está presente nos programas de disciplinas como Psicologia Comunitária, Psicologia Social, Introdução às Ciências Sociais Para a Saúde, Introdução à Antropologia da Saúde, Intervenções em Saúde Coletiva, Psicologia e Políticas Públicas, através do ensino de conteúdos como: metodologias de trabalho que visem a promoção e prevenção de saúde, metodologia qualitativa em saúde, diagnósticos de comunidades e serviços, levantamento e análise de necessidades, visitas domiciliares, pesquisa participante/ação e pesquisa-intervenção.

Já a intervenção como estratégia de organização e mobilização nos movimentos sociais, objetivando transformações sociais, é mencionada em disciplinas como Psicologia Comunitária, Psicologia Social, Psicologia: política e cidadania, Saúde Mental Coletiva, através, por exemplo, dos seguintes conteúdos: possibilidades de ação coletiva na construção de cidadania; análise das diferentes formas de intervenção em ações comunitárias no resgate da cidadania, exclusão, cidadania e saúde;
Psicologia Política. As referências teóricas que fundamentam esses conteúdos, tanto a intervenção como metodologia de pesquisa quanto a intervenção como estratégia de movimento social, são situadas principalmente na perspectiva da Psicologia Social Crítica e também com alguns conceitos teóricos de outras áreas do saber como da Antropologia e da Sociologia.

Quanto ao ensino sobre em quais contextos as intervenções podem ser realizadas pelos psicólogos, destacam-se dois: o da saúde e o de comunidades. $\mathrm{Na}$ área da saúde, podemos visibilizar conteúdos que referem estratégias de ação do psicólogo que visam desde a prevenção até a promoção de saúde e das condições de vida, em programas de disciplinas como Psicologia e Saúde Coletiva, Psicologia e Políticas Públicas, Psicologia da Saúde, Atenção à Saúde, Psicologia Social, Psicologia Comunitária.

Já em relação aos contextos de comunidades, as intervenções são referidas nas questões de pobreza e outras condições de carência na vida e/ou de acesso a serviços, grupos e instituições, estão presentes em disciplinas como: Psicologia Social, Psicologia Comunitária e Psicologia: política e cidadania, a partir de conteúdos como: a inserção do psicólogo no contexto da sociedade brasileira atual; a Psicologia Social em classes desfavorecidas; conceito e vivência da cidadania num contexto de exclusão social; elaboração e avaliação de programas comunitários, entre outros.

Alguns programas de disciplinas, que pretendem o ensino de intervenções sociais como estratégia que discute a melhoria das condições de vida referem a temas relacionados às políticas públicas na área da educação, assistência social, direitos humanos e, principalmente, na área da saúde. Os conteúdos dessas disciplinas que remetem à problematização dessas temáticas apontam para a importância e emergência de políticas públicas e as práticas psi, seja nos direitos humanos, saúde, educação ou assistência social. Porém, esses conteúdos são ainda pouco mencionados estando presentes, especialmente, em programas de disciplinas de Psicologia e Políticas Públicas, Psicologia e Políticas Públicas em Saúde e Psicologia Comunitária. Além disso, no geral, são também pouco evidenciados, em disciplinas dos currículos de Psicologia, conteúdos diretamente ligados ou apresentados de forma sistemática sobre a aprendizagem de políticas públicas e a atuação e trabalho do psicólogo nesta área.

\section{Movimentos Sociais}

Os movimentos sociais enquanto uma temática presente nos conteúdos dos programas das disciplinas da área Psicologia Social e Comunitária remetem, especialmente, a três grandes movimentos: o próprio movimento da Psicologia Social, a Reforma Sanitária e a Reforma Psiquiátrica. 
Os conteúdos das disciplinas desta área trazem, de forma sistemática, o movimento da Psicologia Social, a partir do que foi denominado de "crise da Psicologia Social", situando os contextos da realidade de vida da América Latina e do Brasil e, a partir daí, a inserção de conteúdos sobre outras perspectivas teóricas e metodológicas para a pesquisa e a produção do conhecimento em Psicologia Social. Os programas das disciplinas que mais especificamente tratam deste movimento da Psicologia Social são, em sua maioria, das disciplinas de Psicologia Social e Psicologia Comunitária que apresentam, por exemplo, conteúdos como a definição de Psicologia Social; o âmbito de atuação do psicólogo social; principais contribuições teóricas e temas relativos à Psicologia Social; e Psicologia Social no Brasil e América Latina.

As referências bibliográficas destes conteúdos são nacionais e bastante utilizadas nessas disciplinas, com autores da Psicologia Social Crítica como Sílvia Lane, Pedrinho Guareschi, Wanderlei Codo. Porém, há também referências de autores como Joel Birman e Renato Mezan, que remetem a uma psicanálise com um viés social a partir de conteúdos que problematizam os modos de vida no contemporâneo. Em relação à Psicologia Social norte-americana, o autor referenciado é Aroldo Rodrigues; e quanto à Psicologia Social com enfoque na perspectiva das práticas discursivas e pós-estruturalista, encontram-se Cecília Coimbra, Mary Jane Spink, Neuza Guareschi, entre outros.

Além do conteúdo do movimento social da própria Psicologia Social, nas disciplinas da área da Psicologia Social e Comunitária são apontados de forma menos enfatizada conteúdos que remetem a outros dois movimentos sociais: a Reforma Sanitária e a Reforma Psiquiátrica. Os conteúdos nas disciplinas desta área que se referem a questões da Reforma Sanitária apontam para a história propriamente dita do movimento da saúde que vem eclodir no SUS. Esses conteúdos são apresentados em dois programas e remetem, basicamente, ao fortalecimento do sistema e as práticas do psicólogo no SUS. Estes programas são das disciplinas de Intervenções em Saúde Coletiva e Saúde e dizem respeito à saúde mental coletiva; ao contexto das políticas públicas; as concepções históricas para a compreensão das políticas de saúde; novos paradigmas de saúde comunitária. Os autores que fundamentam estes conteúdos nessas disciplinas são Foucault e outros autores que também são atores de todo esse movimento de Reforma Sanitária como, Gastão Wagner Campos, Roseni Pinheiro, Rubem Mattos.

O movimento da Reforma Psiquiátrica é mencionado mais vezes nos programas das disciplinas desta área. Estes programas apresentam conteúdos a serem ensinados sobre a história deste movimento, especialmente quando a Psicologia Social discute questões de saúde mental e das instituições psiquiátricas. Também são referidos conteúdos nessas disciplinas que tratam de estratégias e ações na saúde mental, a partir da saúde coletiva. Outra questão que emerge a partir dos conteúdos dessas disciplinas de Psicologia Social e Comunitária, quando tratam da Reforma Psiquiátrica, é para refletir a área de saber da Medicina, da Biologia e suas relações com a Psicologia, especialmente, quanto às avaliações psicológicas e diagnósticos, que são referências do psicólogo para o trabalho nessas instituições de saúde mental.

Além dos conteúdos relacionados mais diretamente à Reforma Psiquiátrica e o trabalho do psicólogo nas instituições de saúde mental, os programas das disciplinas de Psicologia Social e Comunitária apontam para a aprendizagem de conteúdos relacionados a outras instituições. Estas instituições são as prisões, casas de internação e abrigagem de crianças e adolescentes, além de outros conteúdos que se referem à exclusão social, à violência e à marginalidade. Podemos identificar, por exemplo, trechos dos programas de disciplinas que tratam de serviços substitutivos da clínica, a partir da reforma psiquiátrica, visando o ensino de outros conceitos e outros temas nesta área. As referências bibliográficas, que fundamentam estes conteúdos sobre as questões da Reforma Psiquiátrica e a discussão mais contemporânea sobre a instituição da saúde mental e o trabalho do psicólogo nesta área, remetem bastante à Psicologia Comunitária, com obras de autores como Regina Campos e Sílvia Lane. Porém, também são propostas referências que trabalham diretamente com a questão da Reforma Psiquiátrica, como Paulo Amarante.

Os conteúdos sobre saúde, a partir do debate da Reforma Sanitária, saúde mental e Reforma Psiquiátrica presentes nas disciplinas da área de Psicologia Social e Comunitária, são aqueles que, no currículo de Psicologia, remetem à outra concepção de saúde ou, ainda, a uma problematização do próprio conceito de saúde, loucura, rede assistencial e cuidado. É interessante apontar que estas questões podem nos fazer pensar que através desses conteúdos que são trabalhados em disciplinas de Psicologia Social e Comunitária está se construindo uma crítica aos conteúdos ensinados em outras disciplinas do currículo de Psicologia, principalmente naquelas que agrupamos nas demais áreas deste estudo - área das Biomédicas e área da Avaliação Psicológica e Psicopatologia; no entanto, não são evidenciado conteúdos ou referências que possam indicar estas relações ou problematizações.

\section{Movimentos dos Currículos de Psicologia em Direção à Formação de Profissionais de Psicologia Para o SUS}

A formação em Psicologia, de acordo com as 
DCNs, deve ter seus currículos direcionados à formação (também) para o SUS. Dessa forma, o olhar que lançamos aos currículos foi este: o de perceber os movimentos que estes vem realizando para dar conta desta proposta de formação. O SUS visibiliza uma visão ampliada do conceito de saúde e, consequentemente, uma visão de sujeito não mais calcada na dicotomia saúde/doença e/ou patologização, mas sim com um viés de promoção de saúde e vida, lançando um olhar integral que abarca os diversos contextos sociais e culturais em que os sujeitos estão inseridos.

Compreendemos que as questões destacadas na discussão do currículo de Psicologia não se esgotam. Contemplamos algumas, mas também consideramos que outras tantas poderiam ser abordadas. No entanto, entendemos que estes pontos que trazemos a seguir nos auxiliam a pensar de que forma está se dando o diálogo da Psicologia com o SUS e, principalmente, de que forma a Psicologia tem se posicionado ética e politicamente na atualidade.

Conforme o princípio da equidade, os sujeitos devem ser respeitados no que se refere às suas individualidades; é a garantia do direito à diferença. Já a integralidade é a afirmativa de que os sujeitos devem ser compreendidos como um todo biopsicossocial. Estabelece-se, assim, a necessidade de um trabalho em equipes transdisciplinares, uma vez que as diversas áreas do conhecimento terão que dialogar para se chegar ao entendimento de atenção em saúde que o SUS propõe. A construção de uma proposta como essa exige a desconstrução das barreiras das especificidades das áreas do conhecimento e o rompimento das fronteiras identitárias.

A preocupação pela promoção das condições de vida, visando buscar possibilidades para as transformações sociais, está nos programas das disciplinas voltadas à Psicologia Social e Comunitária. Embora os objetivos de contrapor as estruturas e relações hegemônicas estejam presentes na proposta curricular das disciplinas dessa área, há diferenças quanto ao tipo de reflexão sobre as origens e efeitos dos diversos contextos sociais, econômicos e culturais e sobre os limites da implicação política das práticas psicológicas sobre os sujeitos. Essas diferenças pautam-se pelos vários enfoques teóricos e metodológicos que, algumas vezes, entram em conflito no posicionamento de temas sobre a ciência psicológica: enquanto algumas abordagens avançam em direção à multidisciplinaridade de conhecimentos, para a compreensão dos objetos de estudo e construção de práticas psi, outras buscam mudanças sociais nas condições de vida dos sujeitos - porém, ainda na crença científica de que deve haver respaldo de saberes e métodos prioritariamente do campo da Psicologia.

Pode-se compreender, de certa forma, que os conteúdos da área de Psicologia Social e Comunitária apontam para a formação do psicólogo em grupos, instituições ou contextos mais amplos da sociedade e menos direcionados ao trabalho do psicólogo junto à saúde pública ou coletiva. E, quando a formação indica estar direcionada à saúde, os conteúdos que evidenciam isto se relacionam mais a saúde do indivíduo, ou seja, mais individualizada e não integrada a análise de outros fatores que possam produzir saúde ou doença. Entretanto, é importante ressaltar que é ainda nessa área da Psicologia Social e Comunitária que se encontram as possibilidades de algumas reflexões teóricas e epistemológicas para inserção e integração da formação em Psicologia na direção do que é proposto pelos princípios do SUS, diferentemente das disciplinas das outras áreas deste estudo, que apresentam muito pouca relação com o campo da saúde coletiva ou para a compreensão do princípio da integralidade e da transformação da concepção de saúde.

Além disso, podemos pensar que a preocupação da Psicologia Social e Comunitária com grupos e populações em desvantagem social, econômica e cultural, propiciou uma aproximação na formação em Psicologia através da inserção de outras perspectivas teóricas e metodológicas que trouxeram um estatuto político na produção do conhecimento na área da Psicologia. Essa aproximação também é a que possibilita alguma formação para que o profissional da Psicologia atue na inserção das práticas psi nas políticas públicas, na construção do exercício da cidadania e nas causas dos Direitos Humanos.

\section{Referências}

Bernardes, J. S. (2006). Políticas Públicas e Formação em Psicologia. EntreLinhas, 33 (7), 4.

Birman, J. (1991). A Physis da Saúde Coletiva. Physis - Revista de Saúde Coletiva, 1 (1), 7-11.

Brasil. Casa Civil (1988). Constituição da República Federativa do Brasil de 1988. Brasília. Acessado de http://www.planalto.gov. br/ccivil 03/constituicao/constitui\%C3\%A7ao.htm.

Brasil. Casa Civil (1990). Lei $n^{\circ} 8.080$, de 19 de setembro de 1990. Brasília. Acessado de http://www.planalto.gov.br/ccivil_03/ Leis/L8080.htm.

Brasil. Conselho Nacional de Educação (2004). Resolução n ${ }^{\circ}$ 8, de 7 de maio de 2004. Brasília. Acessado de http://portal.mec. gov.br/cne/arquivos/pdf/rces08_04.pdf.

Brasil. Ministério da Educação (1996). Lei no 9.394 - de 20 de dezembro de 1996 - DOU de 23/12/96 - Lei Darcy Ribeiro Alterada. Brasília. Acessado de http://www010.dataprev.gov.br/ sislex/paginas/42/1996/9394.htm.

Brasil. Ministério da Educação e Ministério da Saúde (2005). Portaria Interministerial $\mathrm{n}^{\mathrm{o}} 2.118$ de 3 de novembro de 2005. Brasília. Acessado de http://portal.saude.gov.br/portal/arquivos/ pdf/B\%202118\%20INSTITUIR\%20PARCERIA \%20ENTRE\%20 O $\% 20 \% 20 \mathrm{MEC} \% 20 \mathrm{e} \% 20 \mathrm{o} \% 20 \% 20 \mathrm{MS} \% 20 \mathrm{REF}$.pdf.

Brasil. Ministério da Saúde (1986). Relatório Final da $8^{\circ}$ Conferência Nacional de Saúde. Brasília. Acessado de http:// conselho.saude.gov.br/biblioteca/Relatorios/relatorio_8.pdf.

Campos, G. (2006). Políticas de Formação de Pessoal Para o SUS: reflexões fragmentadas. Cadernos RH Saúde, 1 (3), 55-59.

Ceccim, R., \& Carvalho, Y. M. (2005). Ensino da Saúde Como Projeto da Integralidade: a educação dos profissionais de saúde no SUS. In: R. Pinheiro, R. B. Ceccim, \& R. A. Mattos (Orgs.), 
Ensinar Saúde: a integralidade e o SUS nos cursos de graduação na área da saúde (pp. 69-92). Rio de Janeiro: IMS/UERJ: CEPESQ: ABRASCO.

Ceccim, R., \& Feuerwerker, L. C. M. (2004). Mudança na Graduação das Profissões de Saúde Sob o Eixo da Integralidade. Cadernos de Saúde Pública, 5 (20), 1400-1410.

Coimbra, C. (1999). Práticas "psi" no Brasil do "milagre": algumas de suas produções. In: A. M. Jacó-Vilela, F. Jabur e H. B. C. Rodrigues (Orgs.), Clio-Psyquê: histórias da Psicologia no Brasil (pp. 75-91). Rio de Janeiro: Centro Edelstein de Pesquisas Sociais.

Dimenstein, M. (1998). O Psicólogo nas Unidades Básicas de Saúde: desafios para a formação e atuação profissional. Estudos de Psicologia, 1 (3), 53-81.

Farr, R. (1999). As Raízes da Psicologia Social Moderna, (2 ed.). Rio de Janeiro: Vozes.

Foucault, M. (2002). A Psicologia de 1850 a 1950. In: M. Foucault, Ditos e Escritos I - Problematizações do Sujeitos: Psicologia, Psiquiatria e Psicanálise (pp. 220-231). Rio de Janeiro: Forense Universitária.

Hüning, S. M., \& Guareschi, N. M. F. (2009). Efeito Foucault: desacomodar a psicologia. In: N. M. F. Guareschi, S. M. Hüning \& A. A. L. Ferreira (Orgs.), Foucault e a Psicologia, (2 ed., pp. 159182). Porto Alegre: EDIPUCRS

Medeiros, P. F.; Bernardes, A. G., \& Guareschi, N. M. F.
(2005). O Conceito de Saúde e Suas Implicações nas Práticas Psicológicas. Psicologia Teoria e Pesquisa, 21 (3), p. 263-269.

Passos, E. (2001). Por Uma Clínica do Social: relações entre a esfera pública e a esfera privada na Psicologia. Curso de Extensão não publicado. Porto Alegre: Programa de Pós-Graduação em Psicologia da Pontifícia Universidade Católica do Rio Grande do Sul

Passos, E., \& Barros, R. B. (2000). A Construção do Plano da Clínica e o Conceito de Transdisciplinaridade. Psicologia: Teoria e Pesquisa, 16 (1), 71-79.

Silva, R. N. (2005). A Invenção da Psicologia Social. Petrópolis: Vozes.

Spink, M. J. (2003). Psicologia Social e Saúde: práticas, saberes e sentidos. Petrópolis: Vozes.
Recebido: 29/09/2010 Última Revisão: 30/10/2010 Aceite Final: 06/12/2010

Sobre os Autores:

Neuza Maria de Fátima Guareschi - Psicóloga pela PUCRS, mestre em Psicologia Social e da Personalidade pela PUCRS e doutora em Educação pela University of Wisconsin - Madison. Atualmente é professora adjunta da UFRGS, pesquisadora do Programa de Pós Graduação em Psicologia, coordenadora do grupo de pesquisa Estudos Culturais e Modos de Subjetivação e Bolsista Produtividade CNPq.

E-mail: nmguares@gmail.com

Thais Bennemann - Psicóloga pela PUCRS. Atualmente é Residente em Saúde da Família e Comunidade pelo GHC.

E-mail: thais.bennemann@gmail.com

Gisele Dhein: Psicóloga pela UNISC e mestre em Psicologia Social pela PUCRS. Atualmente é professora da UNIVATES.

E-mail: gidhein@gmail.com

Carolina dos Reis - Psicóloga pela PUCRS. Atualmente é Mestranda em Psicologia Social e Institucional pela UFRGS e Assistente de Pesquisa do CRPRS.

E-mail: carolinadosreis@gmail.com

Denise Santos Machry: Psicóloga pela PUCRS. Atualmente é Bolsista Apoio Técnico CNPq pela UFRGS

E-mail: denise.giz.machry@gmail.com 